\title{
Network Coding Aware Queue Management in Multi-Rate Wireless Networks
}

\author{
Nicola De Coppi ${ }^{\dagger}$, Jianxia Ning*, George Papageorgiou*, Michele Zorzi ${ }^{\dagger}$, \\ Srikanth V. Krishnamurthy* and Thomas La Porta \\ ${ }^{\dagger}$ University of Padova \& CFR, Italy ${ }^{*}$ University of California, Riverside, USA ${ }^{\ddagger}$ Pennsylvania State University, USA \\ ${ }^{\dagger}\{$ decoppin,zorzi $\} @$ dei.unipd.it, $\quad *\{$ jning, gpapag, krish $\} @$ cs.ucr.edu, ${ }^{\ddagger}\{t l p\} @$ cse.psu.edu
}

\begin{abstract}
While network coding can potentially provide significant throughput benefits by combining packets prior to forwarding them, the achievable gains are directly related to the coding opportunities at a relay that performs encoding. If the relay does not have packets destined for distinct destinations, that can be encoded together, the network coding gains could be marginal. Towards increasing the opportunities for network coding, in this paper we propose a queue management scheme, that arbitrates the rate at which distinct transmitters send packets to a common relay which applies network coding. Our queue management approach prioritizes the channel access of nodes that do not have enough enqueued packets at the common relay, thereby essentially attempting to balance the number of packets from the distinct senders at the relay. We perform extensive simulations of our approach (built as a wrapper on top of the popular network coding approach COPE) in multi-rate scenarios. We find that our approach yields throughput gains of up to $57 \%$ compared to COPE due to enhanced opportunities towards encoding packets.
\end{abstract}

\section{INTRODUCTION}

Network coding has been proposed to increase the capacity of wireless networks towards the ever increasing demand for wireless capacity due to the emergence of high bandwidth applications. The fundamental idea in network coding is to reduce the number of wireless transmissions by encoding together different packets. In contrast with the traditional store and forward paradigm, network coding uses a store, code and forward approach. Network coding has been applied in both multicast and unicast contexts. In particular, the COPE architecture [1] for network coding in wireless mesh networks has received a lot of attention; COPE has shown that it can improve the throughput of unicast traffic in dense networks with bursty flows. In addition, network coding aware rate adaptation algorithms [2] [3] have recently been proposed; these are built on top of the COPE architecture to further increase throughput.

Scenario of Interest: First, let us revisit network coding in brief. Let us consider the five node topology as in Fig. 1. The five nodes typically occur in larger networks and thus, this topology is often analyzed for its simplicity. In this topology there are two flows, from Alice to Dave and from Bob to Chloe. Jack is the relay node and is responsible for forwarding packets. When COPE is used, Jack transmits an XOR of the packets $(\mathbf{a} \oplus \mathbf{b})$ received from Alice (a) and Bob (b). Using network coding the number of transmissions is reduced from four to three. However, in this example, to decode the coded packet successfully, Chloe and Dave have to correctly overhear packets $\mathbf{a}$ and $\mathbf{b}$, respectively. The radio channel conditions between the sender and the overhearing node, and the transmission rates of Alice and Bob, affect the overhearing at Chloe and Dave. If Chloe cannot overhear the packet from Alice, it will not be able to decode the XORed packet from Jack and the native packet will have to be retransmitted.

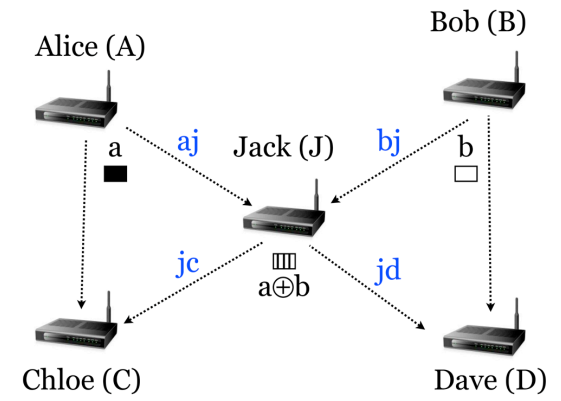

Fig. 1. A topology of five nodes with network coding. Two flows Alice $\rightarrow$ Dave and $\mathrm{Bob} \rightarrow$ Chloe go via Jack who performs network coding.

Loss in Coding Opportunities: When a relay node that performs network coding (Jack) has packets for different next hops (Chloe and Dave), it computes the probability that the receiver nodes can decode the coded packet. If the probability is greater that a certain threshold, it XORs native packets destined for these next hops and sends the coded packet on the wireless channel. However, if the relay does not have packets for the different next hop nodes (Chloe and Dave), it has to send each native packet as they are and thus, the coding opportunity is lost. To visualize the problem, assume that Jack maintains virtual queues for Chloe and Dave. If there is imbalance between these virtual queues, the likelihood that one of these queues becomes empty increases and consequently Jack loses coding opportunities.

Losing coding opportunities can be detrimental to throughput gains possible with network coding and should be avoided, especially in the presence of heavy traffic. Let us again consider our example with Alice, Bob and Jack. If CSMA/CA is the MAC protocol in use, each node in the same Carrier Sense range has the same probability of accessing the channel in the long term. Since the input traffic for Jack is twice its output rate, it starts accumulating packets in the queue. If Jack uses network coding, it drains the queue faster. However, at each instance that Jack loses a coding opportunity, it reduces the rate at which its queue is drained and this contributes to an accumulation of packets in its queue. 
Balancing the aforementioned virtual queues can avoid such losses of coding opportunities. One solution could be to use a perfect schedule wherein Alice and $B o b$ send packets to Jack's queue at equal rates. Jack can explicitly tell Alice and $B o b$ when to transmit. However, Jack may be unaware of whether or not Alice and Bob have packets to transmit; thus a perfect schedule is difficult to implement. Moreover, today most systems use the popular IEEE 802.11 MAC standard. A second simple approach could be for Jack to delay the transmission of a packet until a coding opportunity is created (e.g., Alice's packet is not forwarded unless there is also a packet from $B o b)$. However, this can result in the filling up of Jack's queue and can also cause unnecessary delays; in fact, COPE is built on the underlying principle of never delaying packets.

Our contributions: Based on these considerations, in this paper we propose a queue management approach to increase the probability of coding packets (coding opportunities) in a multi-rate wireless network. Our proposed approach adaptively prioritizes channel access of the transmitters to a relay, based on the states of the virtual queues at the relay. Specifically, the probability of these nodes accessing the channel is adaptively varied by tuning the Contention Window $(C W)$ size at the MAC layer. Adjusting the $C W$ size can be implemented via minor modifications to the IEEE 802.11 standard and has also been used in other contexts (e.g., in [4] and [5]). Based on how many packets are in the virtual queues and the quality of the links, the relay node (Jack) provides the sender nodes (Alice and $B o b$ ) with suggested values of $C W_{\min }$, towards balancing its virtual queues. The value of $C W_{\min }$ is inserted in the header of a COPE packet when such a packet is transmitted by Jack.

The paper is organized as follows. In Section II we present previous studies on network coding and queue management. In Section III we propose our queue management approach. In Section IV we evaluate the performance of our approach via extensive simulations. Section $\mathrm{V}$ concludes our work.

\section{RELATED WORK}

In this section, we describe relevant related work. We first discuss related work on network coding and later that on queue management.

\section{A. Related work on network coding}

Most of the work on network coding in the literature is related to multicast traffic. Network Coding was introduced for the first time by Ahlswelde et al. [6], who showed that routers can achieve multicast capacity by mixing information in different messages. This work was followed by that of $\mathrm{Li}$ et al. who showed that linear codes achieve the maximum capacity bound [7]. They proposed linear network coding where the output at a relay is obtained as a linear combination of its input flows. However, this approach needs centralized knowledge about the network topology. Koetter and Medard proposed random network coding where the linear codes are substituted with codes generated by a polynomial algorithm
[8]. Chou et al. proposed a distributed scheme for pratical network coding which does not need centralized knowledge of the network topology [9].

Katti et al. applied network coding to unicast traffic and integrate network coding into the current network stack [1]. They proposed COPE, a distributed scheme for network coding. With COPE each station is in promiscuous mode to overhear packets which are then stored for a short time $T$ and used for decoding. A relay needs to know which packets its neighbors have in order to be able to code. This information can be sent in periodic reception reports or can be estimated using a routing protocol based on the ETX/ETT metric. COPE by default uses the lowest rate available (i.e., $6 \mathrm{Mbps}$ in IEEE 802.11g). It has been shown in [2] and [3] that network coding aware rate adaptation algorithms can further increase the capacity of wireless networks. None of these efforts however, examine the loss of coding opportunities due to a mismatch in the transmission rates of senders to a common relay. Note that in our work we consider this problem in a multi-rate setting.

\section{B. Related work on queue management}

Most of the queue management algorithms in the literature are proposed to improve congestion control with TCP (e.g., [10], [11]). In the context of network coding, queue models for both unicast [12] and multicast flows have been studied in [13], [14]. Seferoglu et al. propose a queue management algorithm for TCP flows with COPE in [15]. They observe that a mismatch between flow rates can reduce coding opportunities. This mismatch is due to the fluctuation of wireless channel quality. They propose a change to the congestion control mechanisms of TCP to deal with this issue. In particular, when a node is congested, it chooses which packets to drop and the source reduces its packet generation rate. However, their solution does not consider that different flows may have different data rates. In contrast, our approach considers the use of multiple bit-rates in wireless networks; it is also independent of the transport protocol, and thus can be used with both UDP and TCP.

\section{Our Proposed Queue Management ApProACH}

As discussed, ensuring balanced throughput from Alice and Bob can increase Jack's coding opportunities (Fig. 1). We propose to tune the CWs at the senders towards achieving this. We propose a queue management algorithm initiatied by Jack towards estimating the proper $\mathrm{CW}$ sizes for Alice and $B o b$. Jack then feeds back this information to Alice and Bob.

First, let us look at how the queue is managed at the relay node Jack in the COPE architecture. With COPE, each packet enqueued in the output queue at Jack is also enqueued in a virtual queue to the packet's next hop (Chloe or Dave). When Jack senses the channel to be idle, it dequeues the first packet in the output queue and searches the virtual queues to check whether there is a packet with a different next hop, that can be encoded with the dequeued packet (see Fig. 2). The use of virtual queues speeds up the search. 


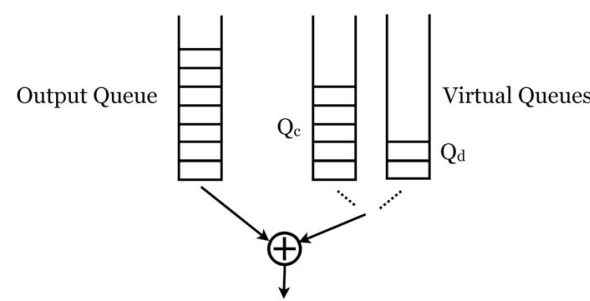

Fig. 2. Output queue and Virtual queues at the relay node for different next hop recipients. $Q_{c}$ and $Q_{d}$ are the sizes of the Virtual queues headed to Chloe and to Dave respectively.

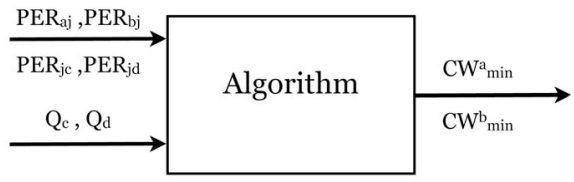

Fig. 3. Block representation of our queue management algorithm.

Having unbalanced virtual queues increases the probability that some of these become empty and thus, cause Jack to miss out on coding opportunities. With our queue management algorithm we want to reduce the probability that a virtual queue becomes empty. We achieve this by appropriately prioritizing the channel access of either Alice or Bob. Based on (i) information on the size of the virtual queues destined to Chloe and Dave ( $Q_{c}, Q_{d}$ in Fig. 2), and (ii) the packet error rates (PERs) on the four links $a j, b j, j c, j d$, (see Fig. 1), our algorithm computes the appropriate $\mathrm{CW}$ sizes for Alice and $B o b$, towards balancing their virtual queues at Jack. In other words, our algorithm takes $Q_{c}, Q_{d}, P E R_{a j}, P E R_{b j}, P E R_{j c}$ and $P E R_{j d}$ as input and outputs the values of $C W_{\text {min }}^{a}$ and $C W_{\text {min }}^{b}$, the contention window sizes for Alice and Bob (see Fig. 3). ${ }^{1}$ Next, we describe the key design elements of our algorithm, and then present the algorithmic details.

The effect of PER on the sizes of the virtual queues: The imbalance in the virtual queues maintained at Jack is primarily because of mismatches in the packet error rates (PER) on the various links in the considered network. Specifically, we make the following observations:

- For the links Alice $\rightarrow$ Jack and Bob $\rightarrow$ Jack, when the medium access is fair as in CSMA/CA and $P E R_{a j}>$ $P E R_{b j}$, Alice has to transmit a packet more times than $B o b$. Thus, at Jack the virtual queue that enqueues packets from Alice will have fewer packets than that from Bob.

- For the receiver links Jack $\rightarrow$ Chloe and Jack $\rightarrow$ Dave, when $P E R_{j c}>P E R_{j d}$, Jack accumulates more packets headed to Chloe (due to a requirement for a higher number of retransmissions).

Tuning $C W_{\min }^{a}$ and $C W_{\min }^{b}$ for balancing queues: By considering the entire path along which packets are delivered (Alice $\rightarrow$ Dave and $\mathrm{Bob} \rightarrow$ Chloe), one can estimate which virtual queue will tend to have fewer packets (at Jack). Based on this, we propose to tune the congestion windows used for

\footnotetext{
${ }^{1}$ The algorithm does not use explicitly any information about the quality of the overhearing links, but we simply assume that this quality is sufficient not to make network coding infeasible.
}

MAC access by Alice and Bob, towards balancing the virtual queues.

We consider the fraction of $\frac{P E R_{\text {receiver }}}{P E R_{\text {sender }}}$. Based on this fraction, we define sender $_{\min }$ and sender $_{\max }$ to be the following:

$$
\begin{gathered}
\text { sender }_{\min } \mid \min \left\{\frac{P E R_{j d}}{P E R_{a j}}, \frac{P E R_{j c}}{P E R_{b j}}\right\} \\
\text { sender }_{\max } \mid \max \left\{\frac{P E R_{j d}}{P E R_{a j}}, \frac{P E R_{j c}}{P E R_{b j}}\right\},
\end{gathered}
$$

where, the notation $\mid$ refers to the sender (Alice or Bob) with the minimum (or maximum) value of the aforementioned fraction. If the virtual queue gets drained faster than the rate at which the packets come into the queue, the queue tends to empty faster. In contrast, if packets arrive to the queue faster than they are drained, the queue tends to fill up. sender $r_{\text {min }}$ is the sender (between Alice and Bob) whose queue tends to fill up (the fraction is typically $<1$ ); sender $_{\max }$ refers to the sender whose virtual queue tends to empty out (the fraction is typically $>1$ ). Thus, our goal is to appropriately increase the probability of packet transmission of sender $\min$ or decrease that of sender $r_{\max }$ so as to balance the virtual queues at Jack.

Consider a link with a packet error rate $P E R_{\text {link }}$. Then, the average number of successful packet transmissions on this link is:

$$
\frac{1}{\left(1-P E R_{\text {link }}\right)} \text {. }
$$

The transmission probability (assuming a fixed contention window $W$ ) is:

$$
p=\frac{2}{(W+1)} .
$$

To increase the rate at which the sender with the higher queue imbalance sender $\min$ sends packets to Jack, we decrease its Contention Window to:

$$
C W_{\min }^{i}=K *\left(C W_{\min }^{j}+1\right)-1
$$

where $i$ is the sender $r_{\text {min }}$ node and $j$ is the other node. $K$ is a coefficient that depends on the PERs of the links on the two paths (defined below). Suppose that Alice is sender $r_{\text {min }}$ and we want to increase her transmission probability while keeping the contention window of Bob (viz. $C W_{\min }^{b}$ ) fixed at 31 (unchanged from default settings). We compute the contention window of Alice to be:

$$
C W_{\min }^{a}=K\left(C W_{\min }^{b}+1\right)-1
$$

where $K=\frac{\left(1-P E R_{a j}\right)\left(1-P E R_{j c}\right)}{\left(1-P E R_{b j}\right)\left(1-P E R_{j d}\right)}$. Note here that $K \leq 1$ since $A$ is sender $_{\text {min }}$, i.e., the contention window size is decreased. This decrease balances the delivery probability of the packets on the two paths Alice $\rightarrow$ Dave and $\mathrm{Bob} \rightarrow$ Chloe and, in turn, balances the virtual queues at Jack.

Alternatively, one can decrease the probability of transmission of $B o b$ by increasing his contention window. We fix the window of Alice to be $C W_{\min }^{a}=31$; we compute $C W_{\min }^{b}$ using (6) except that in lieu of $K$ we use a new coefficient $K^{\prime}=K^{-1} \geq 1$. 
The above solution is used in our queue management algorithm. In particular, when the virtual queues become highly unbalanced, we reduce or increase the contention window. The details of the algorithm are presented later.

Communicating to the sender nodes the value of the congestion window to use : We insert the value of $C W_{\min }$ in the COPE header by adding a new field. Thus, whenever Jack sends a packet to Chloe or Dave, nodes Alice and Bob can overhear the packet by operating in promiscuous mode and extract the suggested minimum $\mathrm{CW}$ values from the header. The overhead for adding the field is negligible; only a few bits per node are needed.

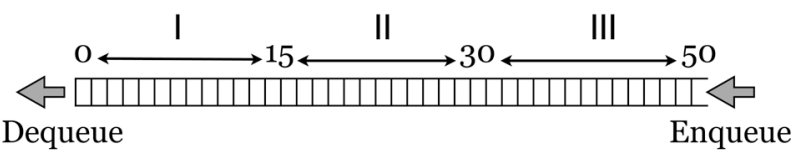

Fig. 4. FIFO output queue divided in three segments.

Details of the algorithm: Recall that our core objective is to ensure that the output queues at the relay node (Jack) have enough packets from both paths to prevent the loss of coding opportunities. Furthermore, the relay node should not have too many enqueued packets; if it does, packets may be dropped resulting in a degradation of the end-toend throughput. Depending on the queue (buffer) capacity, we propose to divide the output queue into three segments as shown in Fig. 4. The example values (15 and 30) are for a queue capacity of 50 packets.

- If the output queue size $\left(Q_{c}+Q_{d}\right) \leq 15$ and the value $\left|Q_{c}-Q_{d}\right|<2$, we increase the transmission probability of the sender $_{\min }$ node as per (6). If the virtual queues become highly unbalanced $\left(\left|Q_{c}-Q_{d}\right| \geq 2\right)$ we divide by 2 the $C W_{\min }$ of the node with fewer packets.

- If the queue size is such that $15<\left(Q_{c}+Q_{d}\right) \leq 30$ and $\left|Q_{c}-Q_{d}\right|<4$,we increase the transmission probability of the sender $r_{\min }$ node as per (6). Otherwise we decrease the transmission probability of the sender $r_{\max }$ node.

- If the queue size is $\left(Q_{c}+Q_{d}\right)>30$, there is a risk that the queue becomes full and begins to drop packets. In this case, we reduce the transmission probability of the node with the higher number of packets: the higher the difference $\left|Q_{c}-Q_{d}\right|$, the higher the $C W_{\min }$ of the node whose queue has more packets. We double the window size until the virtual queue size decreases to below 30 .

In the third case, note that when we reduce the transmission probability of the sender node (Alice or Bob) with the higher number of packets in the queue, Jack has higher probability of accessing the channel and is able to drain his queue faster.

The pseudocode of our queue management algorithm, applied at Jack which is the relay node, is provided in Algorithm 1. The notation [...] under each "else" in the algorithm defines the same actions taken in the corresponding "if" part, in which the roles of $C W_{\min }^{a}$ and $C W_{\min }^{b}$ have been swapped and $\mathrm{K}$ is substituted with $K^{-1}$.

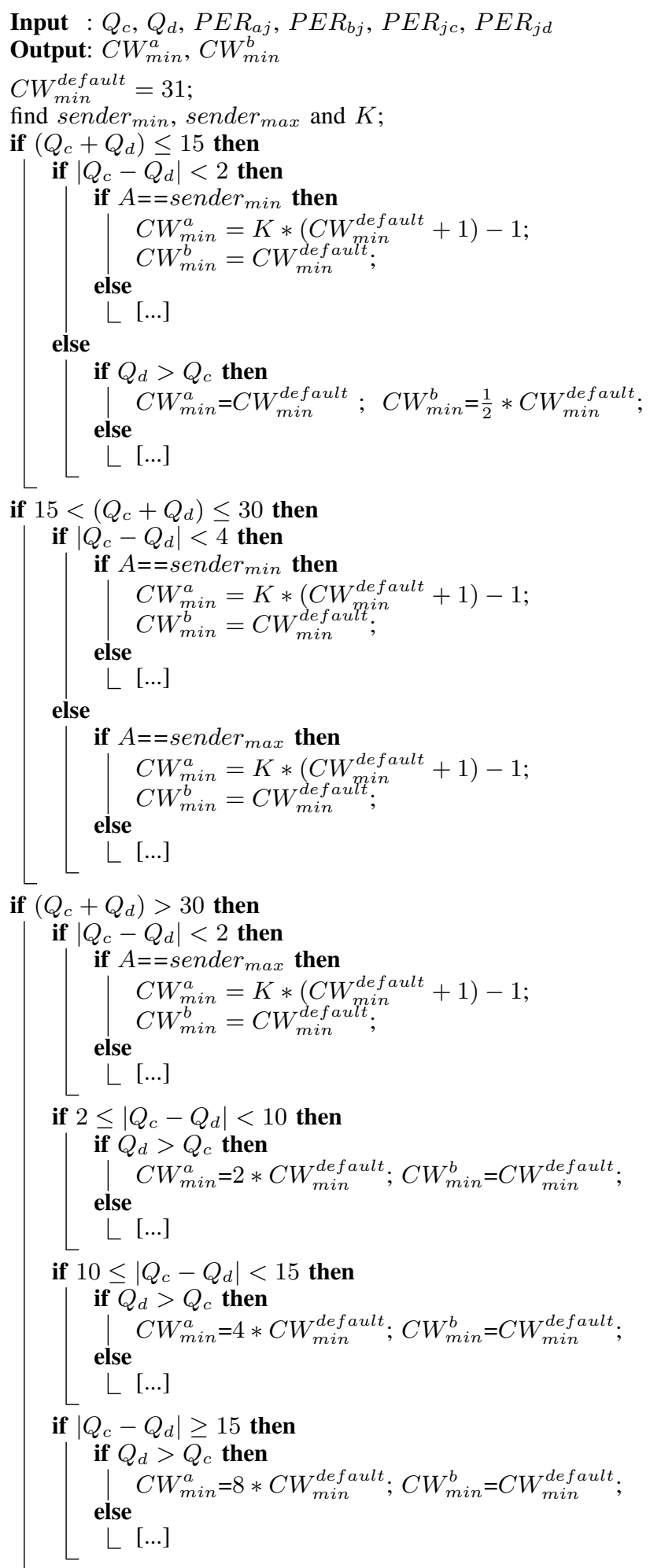

Algorithm 1: Pseudo code of our queue management algorithm. 


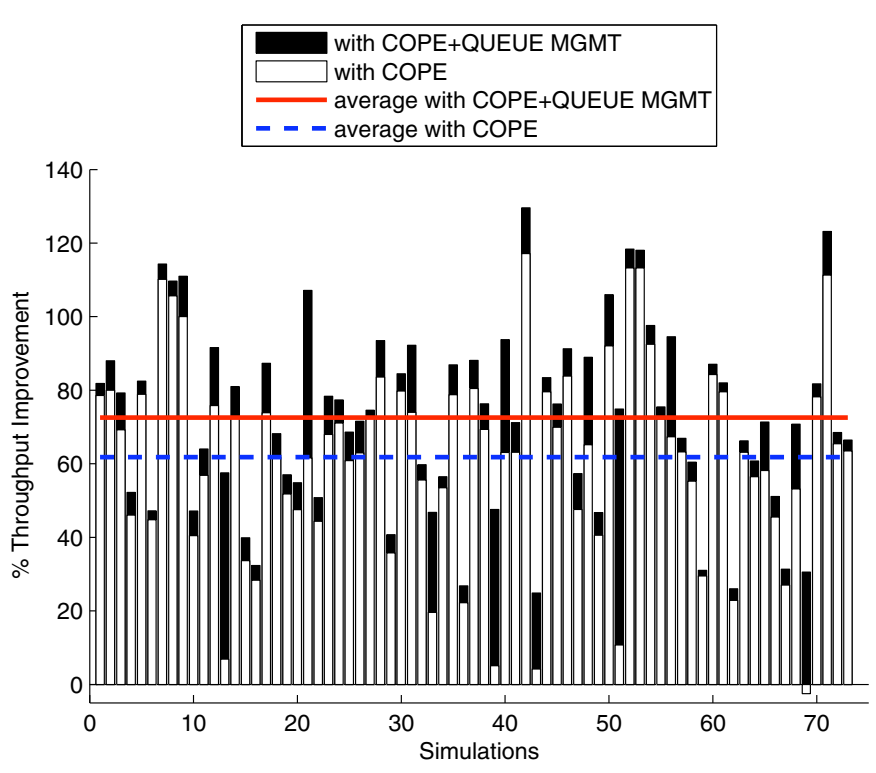

Fig. 5. Throughput gain over a typical IEEE 802.11 system in two cases: with COPE and with COPE + QUEUE MGMT.

\section{Simulation Results}

In this section, we use NS 2.34 [16] simulations to evaluate the proposed queue management algorithm. Since multi-rate transmissions are not supported by NS 2 by default, we used an external library [17]. For the implementation of COPE we began with the Google project [18] and retained the basic structure implemented in the project. We made some modifications and, in particular, changed the coding and decoding processes to exactly conform to the corresponding operations of COPE.

We use IEEE $802.11 \mathrm{~g}$, which allows transmissions at 6,9, 12, 18, 24, 36, 48 and 54 Mbps. Since we deal with static networks, stable Signal-to-Noise Ratios (SNRs) are observed at nodes. The SNR is measured from packets sent on the wireless link; it depends on the distance between two nodes and on the noise power. For the same SNR, transmitting at a lower rate will result in a lower error rate. We use an SNRbased rate adaptation algorithm where each node chooses the highest rate so as to allow all of its neighbors to overhear packets with $P E R \leq 0.2$.

We consider the five node topology in Fig. 1. Two saturated UDP traffic flows are initiated from Alice to Dave and from Bob to Chloe. Each node uses a transmission power of 20 $\mathrm{dBm}$. The $2.4 \mathrm{GHz}$ frequency band is used. The free-space path loss channel model is assumed. The packet size is set to 1500 Bytes. The positions of the nodes are generated randomly and the noise power is randomly chosen from the set $N=\left\{10^{-10}, 2 \times 10^{-10}, 3 \times 10^{-10}, 4 \times 10^{-10}, 5 \times 10^{-10}\right\}$; these values are chosen to be close to the default settings in NS 2. The combination of node positions and noise powers allows us to vary the PER on each link. We consider 73 different combinations. The simulations last for 100 seconds and the results shown are the average over three simulations;

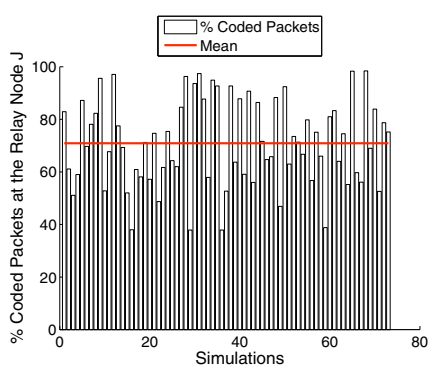

(a)

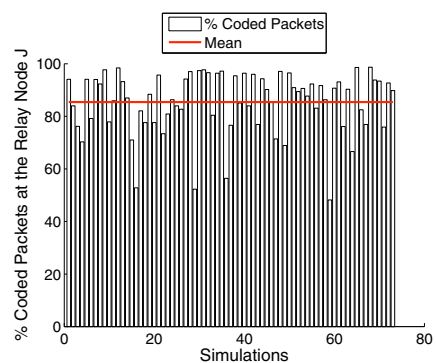

(b)
Fig. 6. Fraction of coded packets over total number of sent packets at the relay node with (a) COPE and (b) COPE + QUEUE MGMT. The average increases from $70 \%$ to $85 \%$.

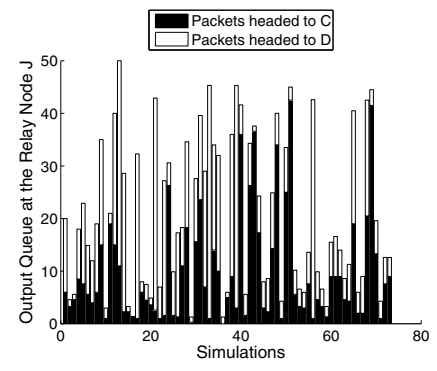

(a)

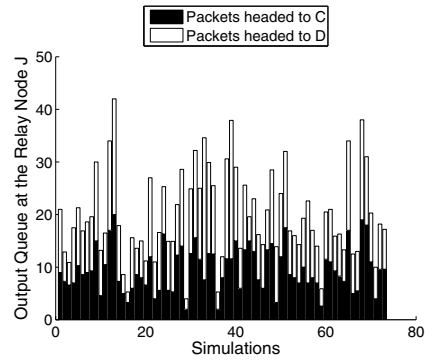

(b)
Fig. 7. Size of the output queue with packets headed to $C$ and $D$ with (a) COPE and (b) COPE + QUEUE MGMT. In the second case the virtual queues are more balanced.

we check the results from the three runs and verify that we have sufficient statistical confidence.

We compare the throughput of the system obtained without COPE, with COPE and with COPE + QUEUE MGMT. Fig. 5 shows the throughput improvements of the system in the 73 different topologies (based on PERs) with COPE and with COPE + QUEUE MGMT with respect to the throughput without COPE. We notice that our algorithm enhances the performance of COPE when COPE's gain is low. On average our algorithm increases COPE's performance by $7.5 \%$, with a maximum improvement of 57\%. Furthermore, the fraction of encoded packets at the relay node increases from $70 \%$ with COPE to 85\% with COPE + QUEUE MGMT (Figs. 6 (a) and (b)). Lastly, the average difference between the virtual queues is 10 packets with COPE and 2.7 packets with COPE + QUEUE MGMT (Figs. 7 (a) and (b)). This shows that our algorithm is able to balance the virtual queues at the relay node and thereby improve coding opportunities.

\section{A. The Best Improvement Case}

First we consider the case where our algorithm provides a system gain of 57\%. This case is shown in Fig. 8. The noise power is fixed to $N=4 \times 10^{-10}$. We observe that the receiver links have different values of PER $\left(P E R_{j c}=0.18\right.$ and $P E R_{j d}=0.009$ ). Packets headed to Chloe, denoted by $C$, will tend to accumulate at the relay node queue (as shown in Fig. 10 (a)). 


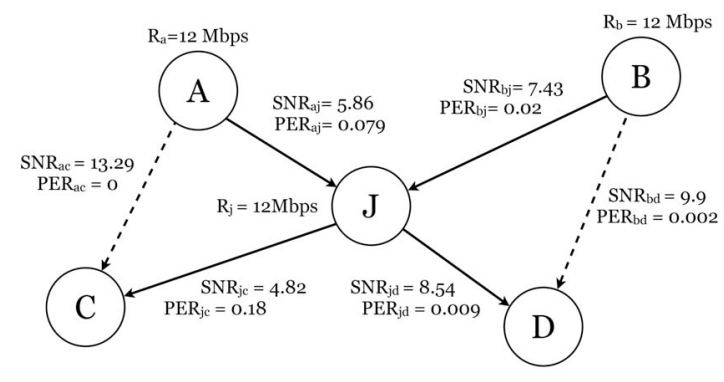

Fig. 8. SNR (in $\mathrm{dB}$ ), data rate and PER for each link in the case where we obtain the best throughput improvement with COPE + QUEUE MGMT.

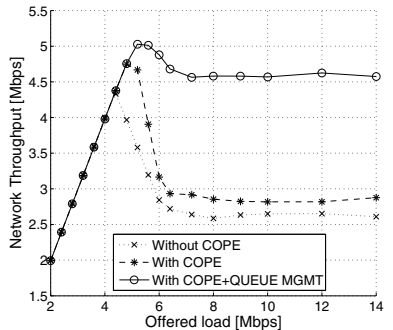

(a)

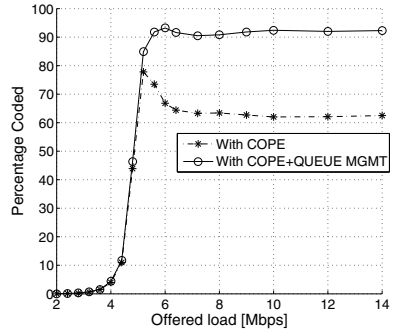

(b)
Fig. 9. (a) COPE+QUEUE MGMT provides $57 \%$ increase in UDP throughput in the presence of saturated traffic. (b) Percentage of packets encoded at the relay node Jack.

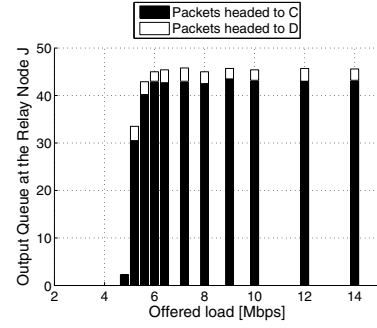

(a)

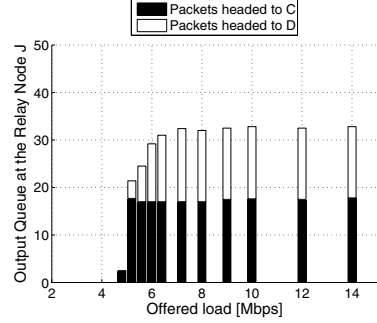

(b)
Fig. 10. Size of the output queue with packets headed to Chloe and Dave (a) with COPE and (b) COPE + QUEUE MGMT. In case (b) the output queue size is reduced and the virtual queues are more balanced.

We gradually increase the offered load in the considered scenario. We see in Fig. 9(a) that the systems have the same throughput as long as the offered traffic is low. At high traffic loads, the performance degrades. However, COPE with our algorithm is able to better cope with the performance degradation by adaptively tuning the transmission probabilities via contention window adjustments. We point out that the fraction of encoded packets is higher with our algorithm (see Fig. 9 (b)). If we look at the output queue at the relay node with only COPE (Fig. 10(a)), we see that the relay node does not accumulate packets in its output queue if the offered load is less than 5 Mbps. After that, the average queue size quickly becomes 45 packets. This means that packets have a high probability of being dropped because the output queue often reaches the maximum size of 50 packets. Moreover, the virtual queues are highly unbalanced. Our algorithm (Fig. 10(b)) reduces the size of the output queue to 30 packets and balances the number of packets headed to different next hop nodes,

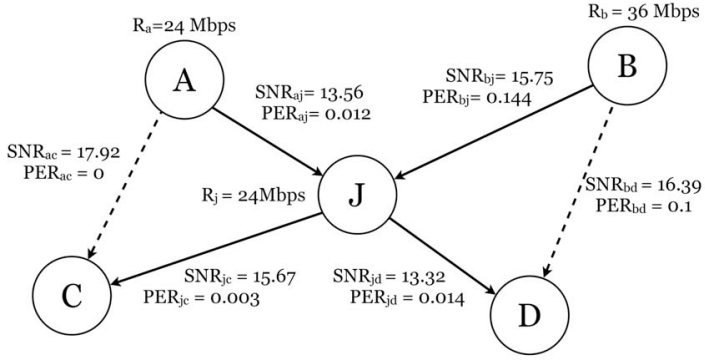

Fig. 11. SNR (in dB), PHY rate and PER for each link in a case where we obtain about a 7\% throughput improvement with COPE+QUEUE MGMT.

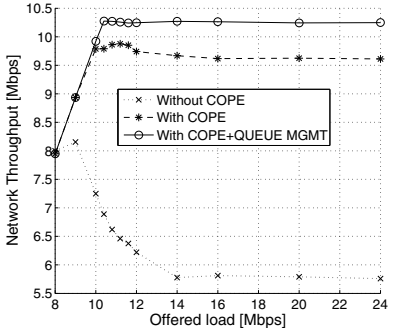

(a)

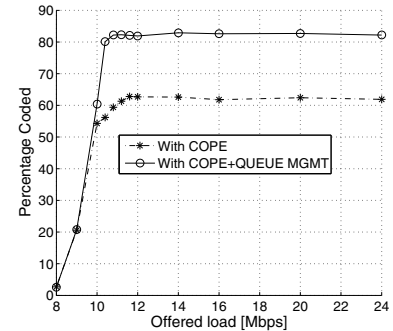

(b)
Fig. 12. (a) COPE+QUEUE MGMT provides $7 \%$ increase in UDP throughput in the presence of saturated traffic. (b) Percentage of packets encoded at the relay node Jack.

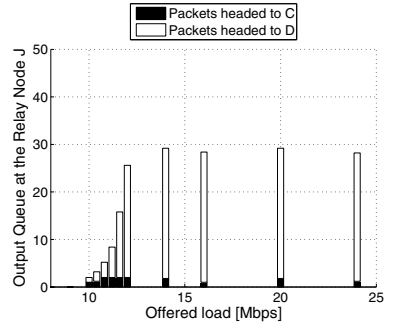

(a)

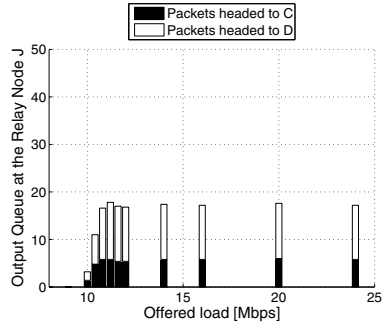

(b)
Fig. 13. Size of the output queue with packets headed to Chloe and Dave with COPE (a) and COPE+QUEUE MGMT (b). In the second case the output queue is reduced and the virtual queu es are more balanced.

thereby increasing coding opportunities at the relay.

\section{B. The Average Improvement Case}

In this case, COPE + QUEUE MGMT provides throughput gains of about $7 \%$ over COPE as seen in Fig. 12(a). Here, we see that there is a difference in the PERs on sender links $\left(P E R_{b j}=0.144\right.$ and $P E R_{a j}=0.012$ as in Fig. 11). In addition, the receiver links have low values of PERs. As seen from Fig. 13(a), without the queue management scheme the relay node accumulates packets headed to Dave, denoted by $D$. This is because of the fact that the relay node receives more packets from Alice (denoted by $A$ ), since $P E R_{a j}<P E R_{b j}$, and thus is able to encode only $60 \%$ of the packets, losing coding opportunities. Again, our algorithm reduces the size of the output queue while making the virtual queues more balanced (Fig. 13(b)). 


\section{CONCLUSIONS}

In this paper we proposed a queue management algorithm on top of the COPE architecture to increase the coding opportunities and network throughput. Our algorithm balances the virtual queues headed to different next hop recipients at a relay node, by adaptively tuning the contention windows of the sender nodes. We simulated our scheme considering a five node topology and the IEEE $802.11 \mathrm{~g}$ standard. We compared the system without COPE, with COPE and with COPE + QUEUE MGMT. Our algorithm achieves a best case throughput gain of 57\% over COPE, with an average gain of $7.5 \%$. As a future work we plan to investigate the scalability of our approach by implementing it and studying the behavior of the relay nodes in a larger network.

\section{ACKNOWLEDGMENTS}

This work was partially supported by the US Army Research Office under the Multi-University Research Initiative (MURI) grant W911NF-07-1-0318, by the NSF NeTS grant 1017012 and by the European Commission under the FP7 EU project SAPHYRE, grant agreement no. 248001.

\section{REFERENCES}

[1] S. Katti, H. Rahul, W. Hu, D. Katabi, M. Medard, and J. Crowcroft, "XORs in the air: Practical wireless network coding," IEEE/ACM Trans. Netw., vol. 16, no. 3, pp. 497-510, Jun. 2008.

[2] T.-S. Kim, S. Vural, I. Broustis, D. Syrivelis, S. Krishnamurthy, and T. La Porta, "A framework for joint network coding and transmission rate control in wireless networks," in Proceedings of INFOCOM, 2010, Mar. 2010.

[3] R. Kumar, S. Tati, F. de Mello, S. Krishnamurthy, and T. La Porta, "Network coding aware rate selection in multi-rate IEEE 802.11," in Proceedings of the $18^{\text {th }}$ IEEE International Conference on Network Protocols (ICNP), 2010, Oct. 2010, pp. 92-102.

[4] A. Nafaa, A. Ksentini, A. Mehaoua, B. 1shibashi, Y. Iraqi, and R. Boutaba, "Sliding contention window (SCW): towards backoff rangebased service differentiation over IEEE 802.11 wireless LAN networks," IEEE Netw., vol. 19, no. 4, pp. 45-51, Jul. 2005.

[5] L. Gannoune and S. Robert, "Dynamic tuning of the contention window minimum (CWmin) for enhanced service differentiation in IEEE 802.11 wireless ad-hoc networks," in Proceedings of the $15^{\text {th }}$ IEEE International Symposium on Personal, Indoor and Mobile Radio Communications, PIMRC 2004, vol. 1, Sep. 2004, pp. 311-317.

[6] R. Ahlswede, N. Cai, S.-Y. Li, and R. Yeung, "Network information flow," IEEE Trans. Inf. Theory, vol. 46, no. 4, pp. 1204-1216, Jul. 2000.

[7] S.-Y. Li, R. Yeung, and N. Cai, "Linear network coding," IEEE Trans. Inf. Theory, vol. 49, no. 2, pp. 371-381, Feb. 2003.

[8] R. Koetter and M. Medard, "An algebraic approach to network coding," IEEE/ACM Trans. Netw., vol. 11, no. 5, pp. 782-795, Oct. 2003.

[9] P. A. Chou, Y. Wu, and K. Jain, "Practical network coding," in Proceedings of Allerton Conference on Communication, Control, and Computing, 2003.

[10] S. Athuraliya, S. Low, V. Li, and Q. Yin, "REM: Active Queue Management," IEEE Netw., vol. 15, no. 3, pp. 48-53, May 2001.

[11] F. W-C., K. Shin, D. Kandlur, and D. Saha, "The BLUE active queue management algorithms," IEEE/ACM Trans. Netw., vol. 10, no. 4, pp. 513-528, Aug. 2002.

[12] S. E. Tajbakhsh, M. Orang, M. H. Sohi, and A. Movaghar, "A queuing model of opportunistic network coding in wireless medium," in International Conference on the Latest Advances in Networks (ICLAN), 2008.

[13] B. Shrader and A. Ephremides, "A queueing model for random linear coding," in In the proceedings of the IEEE Military Communications Conference, MILCOM 2007, Oct. 2007.

[14] M. Iraji, M. Amerimehr, and F. Ashtiani, "A queueing model for wireless tandem network coding," in In the proceedings of the IEEE Wireless Communications and Networking Conferences. WCNC 2009, Apr. 2009.
[15] H. Seferoglu and A. Markopoulou, "Network coding-aware queue management for unicast flows over coded wireless networks," in Proceedings of the IEEE International Symposium on Network Coding (NetCod), 2010, Jun. 2010, pp. 1-6.

[16] The Network Simulator, "ns-2," http://nsnam.isi.edu/nsnam/index.php/ Main_Page.

[17] dei80211mr, "An improved 802.11 implementation for ns2 with enhanced interference model." http://www.dei.unipd.it/wdyn/?IDsezione= 5090 .

[18] COPE on ns2, "Google project by Uppsala University," http://code. google.com/p/uu-cope/. 